\title{
Optimal Strategies for Sending Information through A Quantum Channel
}

\author{
E. Bagan, M. Baig, A. Brey, and R. Muñoz-Tapia \\ Grup de Física Teòrica \& IFAE, Facultat de Ciències, Edifici Cn, Universitat Autònoma de Barcelona, \\ 08193 Bellaterra (Barcelona), Spain \\ R. Tarrach \\ Departament d'Estructura i Constituents de la Matèria, Universitat de Barcelona, \\ Diagonal 647, 08028 Barcelona, Spain
}

(Received 5 July 2000)

\begin{abstract}
Quantum states can be used to encode the information contained in a direction, i.e., in a unit vector. We present the best encoding procedure when the quantum state is made up of $N$ spins (qubits). We find that the quality of this optimal procedure, which we quantify in terms of the fidelity, depends solely on the dimension of the encoding space. We also investigate the use of spatial rotations on a quantum state, which provide a natural and less demanding encoding. In this case we prove that the fidelity is directly related to the largest zeros of the Legendre and Jacobi polynomials. We also discuss our results in terms of the information gain.
\end{abstract}

PACS numbers: 03.67.Hk, 03.65.Bz

One of the problems that is helping us to deepen our understanding of quantum information theory is that of sending information through a quantum channel. Suppose Alice wants to send to Bob the information contained in an arbitrary direction, i.e., in a unit vector $\vec{n}$, which she encodes in a quantum state. This state is sent to Bob, who performs a quantum measurement to retrieve the information stored in the state. Given the characteristics of the information source, and of the quantum channel, there must exist an optimal encoding-decoding procedure which maximizes the knowledge Bob can acquire about $\vec{n}$.

The aim of this Letter is to present such optimal codifications for an isotropic distribution. After considering in detail the lowest dimensions $d=2,3$, and 4, we find the corresponding best procedure for the general case. Such codification, although mathematically very simple, is rather difficult to implement physically. Therefore, we also consider a more natural strategy: that in which Alice performs only physical rotations to her code state [1-4]. In this case we obtain the optimal strategy for any number of spins.

Consider the simplest possible quantum channel, of dimension $d=2$, which can be interpreted as a spin- $1 / 2$ particle. The optimal encoding-decoding procedure is the obvious one [1]: $\vec{n}$ is encoded in the state of spin pointing into $\vec{n}, \vec{\sigma} \cdot \vec{n}|\vec{n}\rangle=|\vec{n}\rangle$, and the decoding is performed by a standard von Neumann spin measurement in an arbitrary direction, $\vec{\sigma} \cdot \vec{m}$. From this measurement, Bob obtains two possible outcomes, \pm 1 , to which he associates the guesses $| \pm \vec{m}\rangle$. We use the fidelity, $(1 \pm \vec{n} \cdot \vec{m}) / 2$, as a figure of merit for Bob's guesses. One could have taken another measure for quantifying the merit of the guess, such as the gain of information (or mutual information), but it complicates substantially the mathematics. Furthermore, previous work [5] seems to indicate that the optimal strategy is insensitive to the use of any of the two figures of merit.
Nevertheless, we present also some comments and results concerning the information gain at the end of this Letter. Now, let us write the average fidelity (for simplicity, we will loosely refer to it simply as fidelity) for $d=2$ as [1]

$$
\begin{aligned}
F^{(2)}= & \int d \vec{n}\left[\frac{1+\vec{n} \cdot \vec{m}}{2}|\langle\vec{n} \mid \vec{m}\rangle|^{2}\right. \\
& \left.+\frac{1-\vec{n} \cdot \vec{m}}{2}|\langle\vec{n} \mid-\vec{m}\rangle|^{2}\right]=\frac{2}{3},
\end{aligned}
$$

where we have used $|\langle\vec{n} \mid \vec{m}\rangle|^{2}=(1+\vec{n} \cdot \vec{m}) / 2$. Notice also that the source, being isotropic, is characterized by the density matrix, $\rho^{(2)}=\int d \vec{n}|\vec{n}\rangle\langle\vec{n}|=I^{(2)} / 2$, where $I^{(d)}$ is the identity in $d$ dimensions, and, hence, has maximal von Neumann entropy, $S\left(\rho^{(2)}\right)=-\operatorname{tr} \rho^{(2)} \log _{2} \rho^{(2)}=1$. This is likely to be a feature of optimal encoding, since the Holevo bound [6], which sets an upper limit on the amount of information accessible to Bob, is precisely $S\left(\rho^{(2)}\right)$ for pure state encoding (recently, it has also been proven that the bound is asymptotically achievable [7]). It is convenient, for what follows, to trade the von Neumann measurement for a continuous (i.e., with infinitely many outcomes) positive operator valued measurement (POVM),

$$
|\vec{m}\rangle\langle\vec{m}|+|-\vec{m}\rangle\left\langle-\vec{m}\left|=2 \int d \vec{m}\right| \vec{m}\right\rangle\langle\vec{m}|=I^{(2)},
$$

which also leads to a maximal fidelity. Notice that this decoding measurement projects on precisely the same states, and with the same relative weights, as those used for encoding the direction. We also recall that for any optimal measurement it is always possible to design a continuous POVM that is also optimal [8]. Therefore, only these need to be considered to find maximal fidelities, although finite measurements leading to the same fidelity can be found [2]. 
Consider now $d=3$, or a spin-1 particle. It is no longer obvious how to encode $\vec{n}$ in an optimal way, since pure states are now characterized by four parameters, while $\vec{n}$ depends only on two. (Recall that the code state, $|A(\vec{n})\rangle \mid$, can be taken to be pure, as if it were a mixed state, one could always replace it with the pure state component which is optimal with respect to the POVM.) In order to determine $|A(\vec{n})\rangle$ we make the following natural assumptions: (a) the optimal code state $|A(\vec{n})\rangle$ is an eigenstate of an operator which can be interpreted as a spin pointing into the direction $\vec{n}$,

$$
\vec{S} \cdot \vec{n}|A(\vec{n})\rangle=S_{n}|A(\vec{n})\rangle, \quad S_{n} \geq 0,
$$

where (b) states corresponding to different directions are related by the "generalized rotations" generated by $\vec{S}$ (these are genuine spatial rotations only if $\vec{S}$ is the total spin of the system). Using this, one can easily solve $d=3$, as there is only one choice for $\vec{S}$ : the spin-1 operators; and only one for $S_{n}: S_{n}=1$ (since $S_{n}=0$ is not one to one). As in the case $d=2$, the source is described by a maximal von Neumann entropy density matrix, $\rho^{(3)}=I^{(3)} / 3, S\left(\rho^{(3)}\right)=\log _{2} 3$. Recall, however, that von Neumann spin measurements are no longer optimal, for it is known that in this case optimal measurements must contain at least four projectors [9]. In fact, no optimal measurement, except for $d=2$, can be of the von Neumann type [9]. It is easy to verify that a continuous POVM, projecting on precisely the code states, $3 \int d \vec{m}|1,1 \vec{m}\rangle\langle 1,1 \vec{m}|=I^{(3)}$, is optimal, where (and hereafter) we use the notation $\vec{S}^{2}\left|S, S_{n} \vec{n}\right\rangle=S(S+1)\left|S, S_{n} \vec{n}\right\rangle$, $\vec{S} \cdot \vec{n}\left|S, S_{n} \vec{n}\right\rangle=S_{n}\left|S, S_{n} \vec{n}\right\rangle$. One finds that the maximal fidelity is

$F^{(3)}=3 \int d \vec{n} \int d \vec{m} \frac{1+\vec{n} \cdot \vec{m}}{2}|\langle 1,1 \vec{n} \mid 1,1 \vec{m}\rangle|^{2}=\frac{3}{4}$.

The problem becomes more complex for $d=4$. There are now two different interpretations of such a Hilbert space: that of a single spin- $3 / 2$ particle or that of two spin- $1 / 2$ particles. Consider first the spin- $3 / 2$ particle interpretation. From (3), we see that either $S_{n}=3 / 2$ or $S_{n}=1 / 2$. The case $S_{n}=3 / 2$ parallels qualitatively that just outlined for $d=3$ and gives for the corresponding optimal measurements $F^{(4)}\left(S=S_{n}=3 / 2\right)=4 / 5$. The choice $S_{n}=1 / 2$ leads to a lower fidelity, in spite of the fact that the two encodings have maximal entropy sources, as for both $\rho^{(4)}=I^{(4)} / 4, S\left(\rho^{(4)}\right)=2$. This can be understood by noticing the following results:

$$
\begin{aligned}
& \left|\left\langle\frac{3}{2}, \frac{3}{2} \vec{n} \mid \frac{3}{2}, \frac{3}{2} \vec{m}\right\rangle\right|^{2}=\left(\frac{1+\vec{n} \cdot \vec{m}}{2}\right)^{3}, \\
& \left|\left\langle\frac{3}{2}, \frac{1}{2} \vec{n} \mid \frac{3}{2}, \frac{1}{2} \vec{m}\right\rangle\right|^{2}=\frac{(1+\vec{n} \cdot \vec{m})(1-3 \vec{n} \cdot \vec{m})^{2}}{8} .
\end{aligned}
$$

Thus, for $S_{n}=3 / 2$, the more $\vec{n}$ differs from $\vec{m}$, the less $\left|\frac{3}{2}, \frac{3}{2} \vec{n}\right\rangle$ and $\left|\frac{3}{2}, \frac{3}{2} \vec{m}\right\rangle$ overlap, i.e., $\left|\left\langle\frac{3}{2}, \frac{3}{2} \vec{n} \mid \frac{3}{2}, \frac{3}{2} \vec{m}\right\rangle\right|^{2}$ is a monotonous function of $(1+\vec{n} \cdot \vec{m}) / 2$, while this is not the case for $S_{n}=1 / 2$. This is a particular instance of a general feature that emerges from our analysis: (c) the overlap of the optimal code states corresponding to different directions, $|\langle A(\vec{n}) \mid A(\vec{m})\rangle|^{2}$, should be a monotonous function of $(1+\vec{n} \cdot \vec{m}) / 2$, ranging from 0 to 1 . The lack of this feature enables us to discard the choice $S_{n}=1 / 2$ without further ado, as we have also verified by explicit computation.

Let us now go to the two spin- $1 / 2$ particle interpretation of $d=4$. Somewhat surprisingly, there are now two possible spin operators. The first is the obvious total spin operator, $\vec{S}=(\vec{\sigma} \otimes I+I \otimes \vec{\sigma}) / 2$, which leads to the Clebsch-Gordan decomposition $\mathbf{1} \oplus \mathbf{0}$. In this case, there are two choices consistent with (3):

$$
\begin{gathered}
|A(\vec{n})\rangle_{1}=|\vec{n}\rangle|\vec{n}\rangle=|1,1 \vec{n}\rangle, \\
|A(\vec{n})\rangle_{0}=\cos \alpha|1,0 \vec{n}\rangle+\sin \alpha e^{i \beta}|0,0\rangle,
\end{gathered}
$$

where $\alpha$ and $\beta$ are $\vec{n}$ independent, as follows from our assumption (b). The $S_{n}=1$ case reduces to the $d=$ 3 one, and gives, of course, the same maximal fidelity (4). For $S_{n}=0$, the overlapping condition (c) implies $\cos \alpha=\sin \alpha=1 / \sqrt{2}$, which we have explicitly checked to be indeed the optimal code for the two spin-1/2 particle interpretation. Notice, however, that the density matrix describing the source no longer has maximal entropy, since now $S\left(\rho^{(3+1)}\right)=1+(1 / 2) \log _{2} 3<2$. This implies that the optimal decoding POVM cannot project on the very same code states, since the corresponding set of projectors has to be a resolution of the identity. Indeed the optimal decoding measurement is given by $4 \int d \vec{m}|B(\vec{m})\rangle\langle B(\vec{m})|=$ $I^{(4)}$, where

$$
|B(\vec{m})\rangle=\frac{\sqrt{3}}{2}|1,0 \vec{m}\rangle+\frac{e^{i \beta}}{2}|0,0\rangle,
$$

and gives a fidelity

$$
\begin{aligned}
F^{(3+1)} & =4 \int d \vec{n} \frac{1+\vec{n} \cdot \vec{z}}{2}|\langle A(\vec{n}) \mid B(\vec{z})\rangle|^{2} \\
& =\frac{3+\sqrt{3}}{6},
\end{aligned}
$$

where $\vec{z}$ is the unit vector pointing in the $z$ direction and rotational invariance enabled us to integrate $\vec{m}$ trivially. Notice that $e^{i \beta}= \pm 1$ corresponds to the code states chosen by Gisin and Popescu [3] that led them to the conclusion that antiparallel spins encode information about $\vec{n}$ more efficiently than parallel spins. Our result reproduces theirs, which was later proven to be optimal [4]. Note, however, that the fidelity (10) is lower than $4 / 5$, the spin- $3 / 2$ particle interpretation result.

Before discussing our results, let us dispose of the other spin operators, which are in fact a one-parameter family, $\quad S_{i}=\left(\cos ^{2} \eta \sigma_{i} \otimes I+\sin ^{2} \eta I \otimes \sigma_{i}+\sin \eta \times\right.$ $\left.\cos \eta \sum_{j, k} \epsilon_{i j k} \sigma_{j} \otimes \sigma_{k}\right) / 2$. They generate the $\mathbf{1} / \mathbf{2} \oplus \mathbf{1} / \mathbf{2}$ 
representation, and one can easily check that $F^{(2+2)}=$ $F^{(2)}$. It is thus of no interest.

We can draw the following conclusion from our analysis of $d=4$ : the optimal encoding is given by the spin-3/2 interpretation, i.e., by the only encoding which satisfies Eq. (3), the overlapping condition (c), and corresponds to a maximal entropy source. This is, after all, what one would have expected. This result can be generalized to an arbitrary dimension: the single spin- $(d-1) / 2$ interpretation of a $d$-dimensional Hilbert space gives the optimal encoding with maximal fidelity

$$
F^{(d)}=\frac{d}{d+1} .
$$

If $d=2^{N}$, one can, of course, perform this optimal encoding with $N$ spin- $1 / 2$ particles (qubits).

Let us now illustrate this for the simple case of two qubits: the operators $S_{i}$ corresponding to the spin-3/2 interpretation can be written as [10] $S_{x}=(\sqrt{3} / 2) I \otimes$ $\sigma_{x}+\left(\sigma_{x} \otimes \sigma_{x}+\sigma_{y} \otimes \sigma_{y}\right) / 2, S_{y}=(\sqrt{3} / 2) I \otimes \sigma_{y}+$ $\left(\sigma_{y} \otimes \sigma_{x}-\sigma_{x} \otimes \sigma_{y}\right) / 2$, and $S_{z}=(1 / 2) I \otimes \sigma_{z}+$ $\sigma_{z} \otimes I$. These operators fulfill the $\mathrm{SU}(2)$ algebra, $\left[S_{i}, S_{j}\right]=i \epsilon_{i j k} S_{k}$, but they are not the components of a vector under spatial rotations, generated by the total spin of the two particles (we have already found that the only vector representations are $\mathbf{1} \oplus \mathbf{0}$ and $\mathbf{1 / 2} \oplus \mathbf{1 / 2}$ ). The unitary transformations generated by these operators are nonlocal and difficult to implement physically. Furthermore, they can change the entanglement of the states. For instance, the product state $\left|\frac{1}{2}, \frac{1}{2} \vec{z}\right\rangle \otimes\left|\frac{1}{2}, \frac{1}{2} \vec{z}\right\rangle$, which is an eigenvector of $S_{z}$, becomes entangled under the transformation $e^{i \theta S_{y}}$ for $\theta=\pi / 2$ but remains a product state for $\theta=\pi$. The optimal decoding can be achieved by a continuous POVM, but there are finite POVM's that are optimal, too [2,9]. For example, from Ref. [9] one can read off that the minimal optimal POVM corresponds to six equally weighted projectors associated to the six unit vectors pointing at the vertices of a regular octahedron.

The merit of the procedure just outlined is, obviously, that the maximum possible value of the fidelity is attained. However, the encoding process, involving complicated unitary operations, looks exceedingly demanding. It is therefore important to examine a less contrived method in which Alice can perform only spatial rotations on an initial code state: she may, e.g., rotate the device that produces her initial states. This is, actually, the approach followed in $[1,2]$ for parallel spin code states, where the maximum fidelity in terms of the number of spins was found to be $F=(N+1) /(N+2)$, and in [3,4] for two antiparallel spins. In fact, for two spins we have already found that the family of states (8) with $\alpha=\pi / 4$ (to which the two antiparallel spin states of [3,4] belong) is indeed the best Alice can use if she is allowed to perform only space rotations. We now generalize this physically more feasible strategy to any number of spins and calculate its maximal fidelity.
Let us sketch the main steps of the calculation (a more detailed discussion will be presented elsewhere [11]). First, one considers, as usual, continuous POVM's for decoding. Second, note that according to the ClebschGordan decomposition, any state of $N$ spin- $1 / 2$ particles can be written as a combination of states $\left|S, S_{n} \vec{n}\right\rangle, \quad 0 \leq S \leq N / 2$, belonging to the irreducible representation $\mathbf{S}$ (here $S, S_{n}$ obviously refer to the total spin operator), where $\mathbf{S}$ usually appears more than once for $S<N / 2$. Third, one notices that these repeated representations do not add any further knowledge about $\vec{n}$, hence, the Hilbert space, $\mathcal{H}$, of the code states can be chosen to be

$$
\mathcal{H}=\frac{\mathbf{N}}{\mathbf{2}} \oplus\left(\frac{\mathbf{N}}{\mathbf{2}}-\mathbf{1}\right) \oplus\left(\frac{\mathbf{N}}{\mathbf{2}}-\mathbf{2}\right)+\cdots .
$$

States living in more than one equivalent representation can also be used, but this just complicates the computation and leads to the same maximal fidelity. According to Eqs. (3) and (12), the optimal code state can be written as $|A(\vec{n})\rangle=\sum_{S=S_{n}}^{N / 2} A_{S}\left|S, S_{n} \vec{n}\right\rangle$, where $\sum_{S=S_{n}}^{N / 2}\left|A_{S}\right|^{2}=1$. One must choose the minimal possible value of $S_{n}$, that is, $S_{n}=0$ if $N$ is even, and $S_{n}=1 / 2$ if $N$ is odd, since these choices use the largest available dimension of the code state space (12) [11]. The explicit calculation of the fidelity function corresponding to the optimal POVM, for which $|B(\vec{m})\rangle$ is a straightforward generalization of (9), leads to

$$
F=\frac{1}{2}+\frac{1}{2} \mathrm{~A}^{t} \mathrm{MA}
$$

where $\mathrm{M}$ is a matrix of tridiagonal form

$$
\mathbf{M}=\left(\begin{array}{ccccc}
d_{l} & c_{l-1} & & 0 \\
c_{l-1} & \ddots & \ddots & 0 & \\
& \ddots & d_{3} & c_{2} & \\
& & c_{2} & d_{2} & c_{1} \\
0 & & & c_{1} & d_{1}
\end{array}\right)
$$

that can be chosen to be real. Here

$$
l=N / 2+1-S_{n},
$$

and $\mathrm{A}^{t}=\left(\left|A_{N / 2}\right|,\left|A_{N / 2-1}\right|,\left|A_{N / 2-2}\right|, \ldots\right)$, where $\mathrm{A}^{t}$ is the transpose of $\mathrm{A}$. If $N$ is even, the coefficients of $\mathrm{M}$ are $d_{k}=0, c_{k}=k / \sqrt{4 k^{2}-1}$; otherwise, if $N$ is odd, $d_{k}=1 /\left(4 k^{2}-1\right), \quad c_{k}=\sqrt{k(k+1)} /(2 k+1) . \quad$ The largest eigenvalue, $x_{l}$, of $\mathrm{M}$ determines the maximal fidelity through the relation $F=\left(1+x_{l}\right) / 2$. To find $x_{l}$, we set up a recursion relation for the characteristic polynomial of $\mathrm{M}$ :

$$
Q_{l}(x)=\left(d_{l}-x\right) Q_{l-1}(x)-c_{l-1}^{2} Q_{l-2}(x) .
$$

We are now at the end of the calculation, as the solutions of (16) are just proportional to the Legendre polynomials, $P_{l}(x)$, if $N$ is even, and to the Jacobi polynomials [12], 
TABLE I. Maximum fidelities in terms of the number of spins for space rotations

\begin{tabular}{cccccccc}
\hline \hline$N$ & 1 & 2 & 3 & 4 & 5 & 6 & 7 \\
\hline$F$ & $\frac{2}{3}$ & $\frac{3+\sqrt{3}}{6}$ & $\frac{6+\sqrt{6}}{10}$ & $\frac{5+\sqrt{15}}{10}$ & 0.9114 & 0.9306 & 0.9429 \\
\hline \hline
\end{tabular}

$P_{l}^{0,1}(x)$, if $N$ is odd. The eigenvalue $x_{l}$ is precisely the largest zero of the corresponding polynomial.

The values of the maximal fidelity for $N$ up to seven are collected in Table I. Notice that the optimal encoding for three spins gives $F=(6+\sqrt{6}) / 10 \sim 0.845$, which is a better result than the optimal value for four parallel spins $(F=5 / 6 \sim 0.833[1])$. In fact, it can be shown that our maximal fidelity approaches unity quadratically in the number of spins:

$$
F \sim 1-\frac{\xi^{2}}{N^{2}}
$$

where $\xi \sim 2.4$ is the first zero of the Bessel function $J_{0}(x)$, while for $N$ parallel spins the fidelity approaches unity only linearly: $F \sim 1-1 / N$. At this point, we feel compelled to go back to (11) and point out that for the optimal encoding, based on generalized rotations, the fidelity tends exponentially to unity: $F \sim 1-2^{-N}$.

Up to now we have restricted ourselves to finding optimal strategies using the fidelity to quantify the quality of the encodings. We conclude by making a few comments on their quantum information gain. We, therefore, work out this quantity for the optimal strategies that led to (11).

For a continuous POVM the symmetry of the problem enables us to simplify the computation, as only the contribution of a single projector is needed (say the one in the $\vec{z}$ direction). After canceling the divergent terms associated to the continuous distribution of the code states $|A(\vec{n})\rangle$, the average information gain is just [11]

$$
\begin{aligned}
I_{\mathrm{av}}= & \int d \vec{n}\left(d|\langle A(\vec{n}) \mid B(\vec{z})\rangle|^{2}\right) \\
& \times \log _{2}\left(d|\langle A(\vec{n}) \mid B(\vec{z})\rangle|^{2}\right),
\end{aligned}
$$

where $|B(\vec{z})\rangle=|S, S \vec{z}\rangle$, and $d$, the dimension of the code state space, is related to $S$ by $d=2 S+1$. In terms of $d$ one obtains $I_{\mathrm{av}}=\log _{2} d-(1-1 / d) \log _{2} e$, a result also found in [5]. In terms of $N$, it reads

$$
I_{\mathrm{av}}=N-\left(1-2^{-N}\right) \log _{2} e .
$$

This is just the number of qubits transmitted in the process, minus a term that asymptotically goes to a constant.
Finally, it is interesting to study the information gain using the simpler, but not truly optimal, encoding. For $N=2$, the best code state according to the fidelity is given by (8) with $\alpha / \pi=1 / 4$ (maximal fidelity and information gain are both independent of $\beta$ ). The information gain is $I_{\mathrm{av}}=0.8664$, less than that obtained applying the optimal encoding for which (19) gives $I_{\mathrm{av}}=0.9180$. Nevertheless, we could ask ourselves if this gain is maximal for code states of the form (8). An explicit computation shows that this is not so, as the maximal gain is $I_{\mathrm{av}}=0.8729$ for $\alpha / \pi=0.2317 \neq 1 / 4$. Hence, at least in this case, states with maximal fidelity and maximal information gain do not coincide; they seem to do so only when the truly optimal strategy is considered.

To summarize, we have presented optimal encodingdecoding procedures for sending the information contained in an arbitrary direction faithfully codified in a quantum state. For restricted encodings, based on space rotations, the maximal fidelity is related to the largest zeros of the Legendre or Jacobi polynomials. Although this encoding does not make full use of the quantum channel capacity, our results show a significant improvement over previous strategies based on parallel spin encoding.

We thank S. Popescu, A. Bramon, G. Vidal, and W. Dür for stimulating discussions and M. Lavelle for reading the manuscript. Financial support from CICYT Contracts No. AEN98-0431 and No. AEN99-0766, CIRIT Contracts No. 1998SGR-00026, No. 1998SGR-00051, and No. 1999SGR-00097, and EC Contract No. IST1999-11053 is acknowledged.

[1] S. Massar and S. Popescu, Phys. Rev. Lett. 74, 1259 (1995).

[2] R. Derka, V. Buzek, and A. K. Ekert, Phys. Rev. Lett. 80, 1571 (1998).

[3] N. Gisin and S. Popescu, Phys. Rev. Lett. 83, 432 (1999).

[4] S. Massar, Phys. Rev. A 62, 040101(R) (2000).

[5] R. Tarrach and G. Vidal, Phys. Rev. A 60, R3339 (1999).

[6] A. S. Holevo, Probl. Inf. Transm. (Engl. Transl.) 9, 177 (1973).

[7] A.S. Holevo, IEEE Trans. Inf. Theory 44, 269 (1998); B. Schumacher and M. Westmoreland, Phys. Rev. A 56, 131 (1997).

[8] A.S. Holevo, Probabilistic and Statistical Aspects of Quantum Theory (North-Holland, Amsterdam, 1982).

[9] J. I. Latorre, P. Pascual, and R. Tarrach, Phys. Rev. Lett. 81, 1351 (1998).

[10] A. Peres, Quantum Theory: Concepts and Methods (Kluwer, Dordrecht, The Netherlands, 1995).

[11] E. Bagan et al. (to be published).

[12] For standard definitions and conventions, see, for instance, M. Abramowitz and I. A. Stegun, Handbook of Mathematical Functions (Dover, New York, 1970). 